\title{
PUBIS STRESS FRACTURE IN A 15-YEAR-OLD SOCCER PLAYER
}

Fabrício Melo Bertolini', Rodrigo Barreiros Vieira², Lucas Henrique Araujo de Oliveira ${ }^{3}$, Rodrigo Pace Lasmar ${ }^{4}$, Otaviano de Oliveira Junior ${ }^{2}$

\section{ABSTRACT}

This case report presents a 15-year-old football player who was diagnosed with a pubis stress fracture and underwent conservative treatment with satisfactory results. After a review of the literature, the clinical picture, diagnosis and

\section{INTRODUCTION}

Stress fractures are a very common phenomenon in sports medicine. It is believed that they occur in athletes mainly because of repeated microtrauma and continual muscle tensioning over the bone ${ }^{(1,2)}$. The records indicate that stress fractures account for up to $20 \%$ of all sports injuries and around $4.7 \%$ to $15.6 \%$ in runners ${ }^{(3)}$. Most stress fractures occur in the lower limbs, and the tibia, distal fibula and metatarsals are the commonest fracture sites ${ }^{(2)}$. However, stress fractures of the public bone are rare and there are very few descriptions in the literature. They are considered to present low risk and have been observed to have greatest incidence among long-distance runners and female military recruits ${ }^{(2)}$. The present case relates to a 15 -year-old male athlete belonging to a soccer club in Belo Horizonte who suffered a stress fracture of the right superior pubic ramus and underwent conservative treatment with satisfactory regeneration of the injured structure. The lack of reports on this fracture in the medical-scientific literature, treatment are described. The importance of this paper comes from the rarity of finding reports about this kind of injury in the literature.

Keywords - Fractures, Stress; Osteitis/physiopathology; Pubic Bone specifically in relation to soccer players, was our motivation for producing this study.

\section{CASE REPORT}

The patient was a 15-year-old male who was a soccer player at Atlético Mineiro Soccer Club.

He complained of pain in his proximal right thigh, on the medial face, which began insidiously after a soccer match, without any history of local trauma. He did not present any previous bone-muscle injuries. On examination on that day, he felt pain on palpation at the origin of the adductors of the right thigh, and on adduction of the leg against resistance. Thus, his condition was initially diagnosed as adductor tendinitis. He was taken off his physical activities and analgesic measures were started, with the use of NSAIDs and physiotherapy for four weeks. After this period, he was seen to be asymptomatic and pain-free at the physical reevaluation, and was allowed to return to sports activities without restrictions.

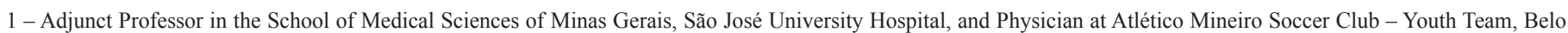
Horizonte, Brazil.

2 - Orthopedist and Traumatologist; Head of the Medical Department, Atlético Mineiro Soccer Club, Belo Horizonte, Brazil.

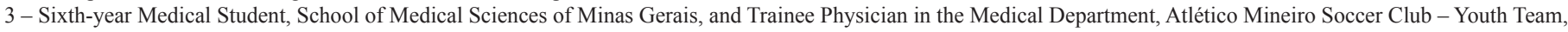

Belo Horizonte, Brazil.

4 - Orthopedist and Traumatologist; Physician at Atlético Mineiro Soccer Club - Youth Team, Belo Horizonte, Brazil.

Work performed in the Medical Department, Atlético Mineiro Soccer Club - Youth Team.

Correspondence: Rua Palmira, 528, apto. 208 - 30220-110 - Belo Horizonte, MG - E-mail: fabriciobertolini@hotmail.com

Work received for publication: June 04, 2010; accepted for publication: August 23, 2010.

The authors declare that there was no conflict of interest in conducting this work 
Three days later, the patient again started to feel mild pain at the proximal insertion of the adductors of the right thigh during running training and, by the end of the activity, he presented difficulty in walking. On examination, he felt pain on palpation in the tendinous region of the adductors, and said that the pain was worse in adduction tests on the right thigh performed passively, actively and against resistance, He was bilaterally positive in the Grava test ${ }^{(4)}$.

With the need to clarify the hypothesis of pubic osteitis, magnetic resonance imaging (MRI) was requested. The athlete was again taken off his physical activities and referred for physiotherapy to start treatment with anti-inflammatory and analgesic measures, by means of electrotherapy and cryotherapy. The MRI examination showed a stress fracture of the right superior pubic ramus, accompanied by significant edema, and a mature bone callus in the anterior/lower third of the left inferior pubic ramus, with slight remaining edema, as can be seen in Figures 1 and 2 respectively. The conservative treatment with physiotherapy was continued.

Twelve weeks later, the athlete was found to be asymptomatic and was sent for MRI again. This showed that the right-side fracture and left pubic bone edema still persisted (Figures 3 and 4).

Anteroposterior radiographs of the pelvis and bone scintigraphy were also requested, and these can be seen in Figures 5 and 6. These examinations demonstrated deformity of the right pubis without soft-tissue abnormalities, along with slight uptake of the radiopharmaceutical in the pubis, suggestive of bone remodeling.

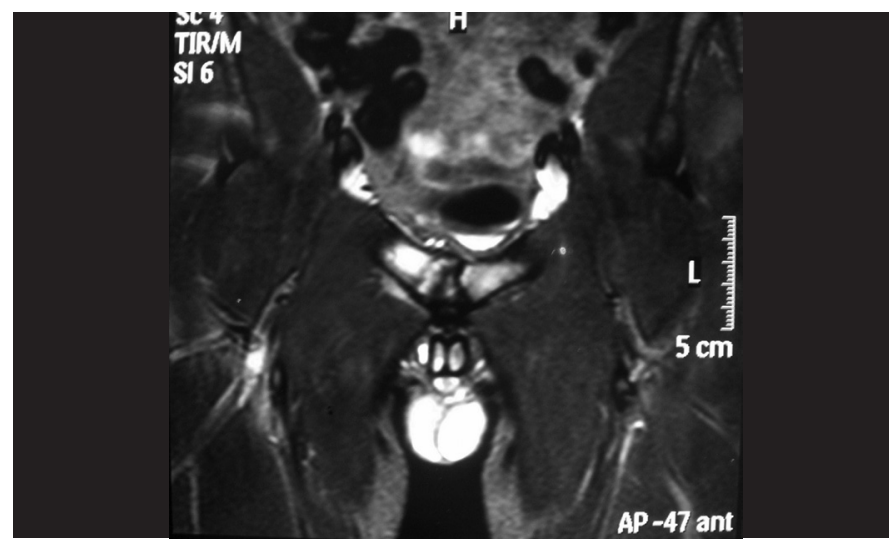

Figure 1 - Magnetic resonance imaging showing differentiated linear fracture line within the spongy bone in the right superior pubic ramus, adjacent to the pubic symphysis, accompanied by significant bone edema extending to the common origin of the adductors.

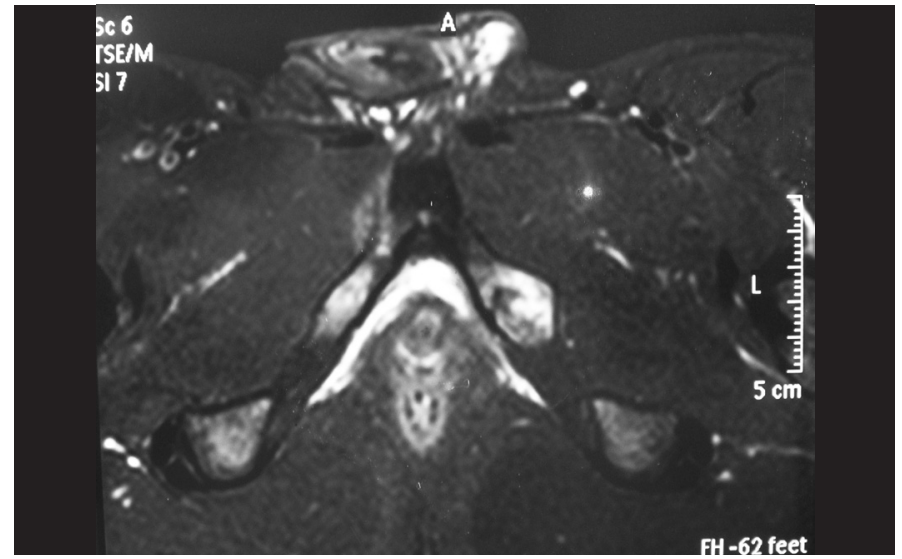

Figure 2 - Magnetic resonance imaging showing bone callus with slight edema in the contiguous margins, located in the anterior and middle thirds of the left inferior pubic ramus.

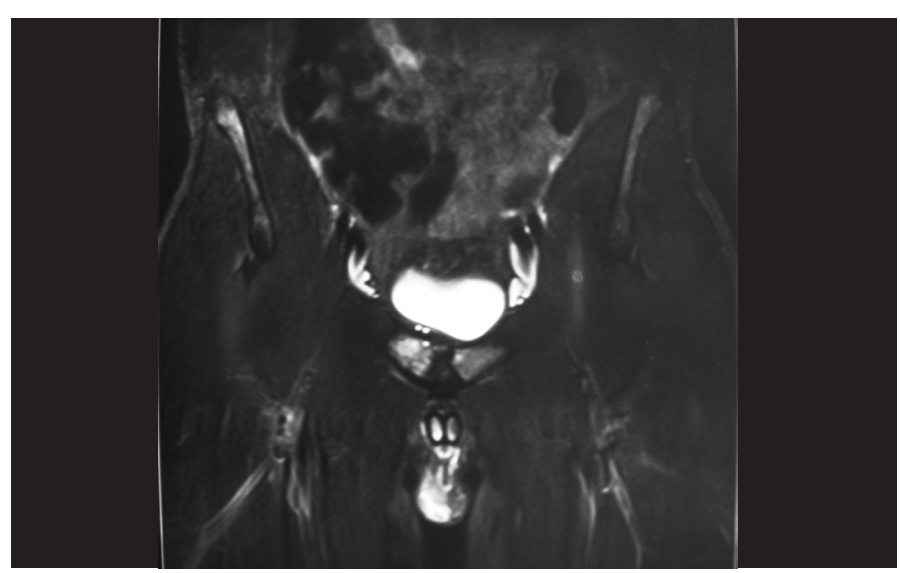

Figure 3 - Magnetic resonance imaging showing line of vertical fracture in the body of the right pubic bone, without reaching the joint surface and with edema in the adjacent bone marrow.

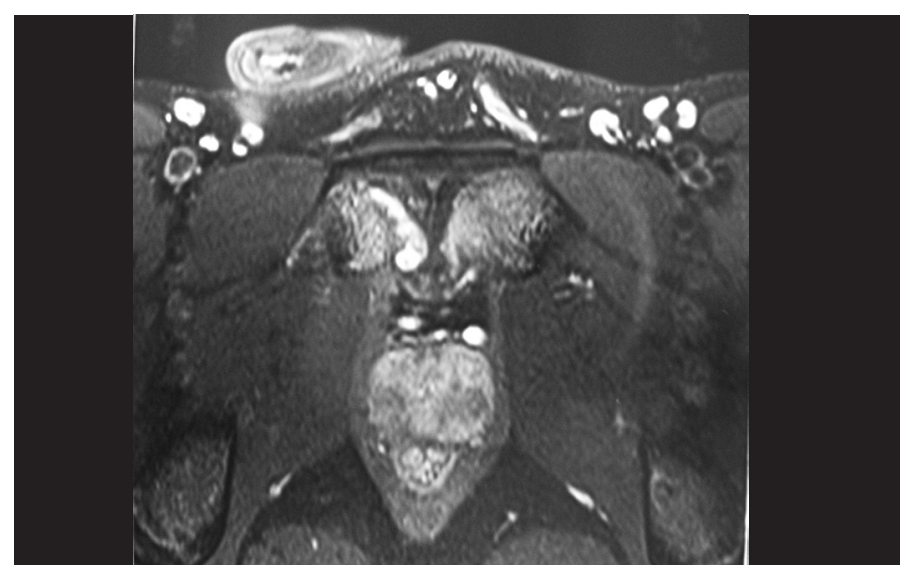

Figure 4 - Magnetic resonance imaging showing slight bone marrow edema in the body of the left public bone.

The athlete continued to undergo physiotherapy, with muscle rebalancing exercises to stabilize the hips (focusing on the pubis) applied to the adductor and abdominal musculature. During this phase, concentric abdominal exercises were performed first, and 


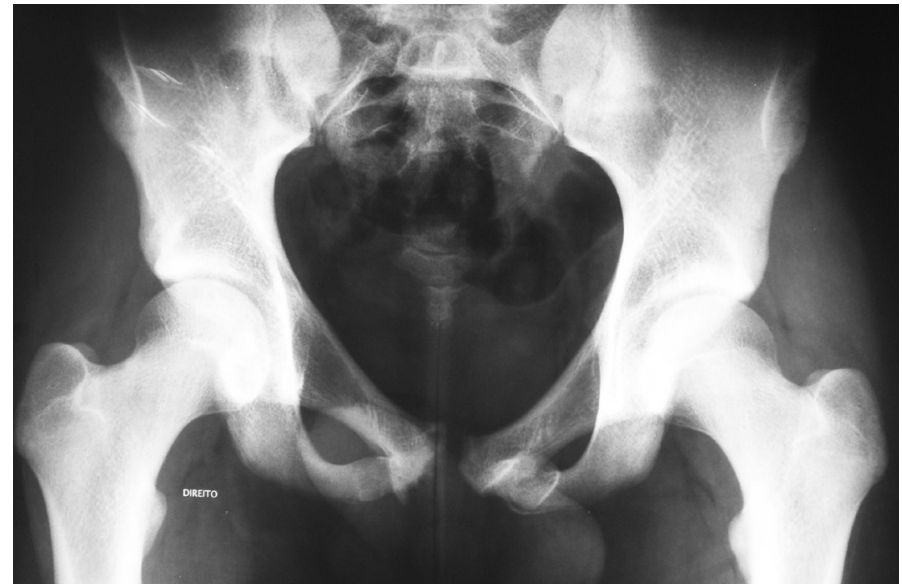

Figure 5 - Radiograph on the pelvis, showing deformity of the right pubic bone with widening of the joint space.

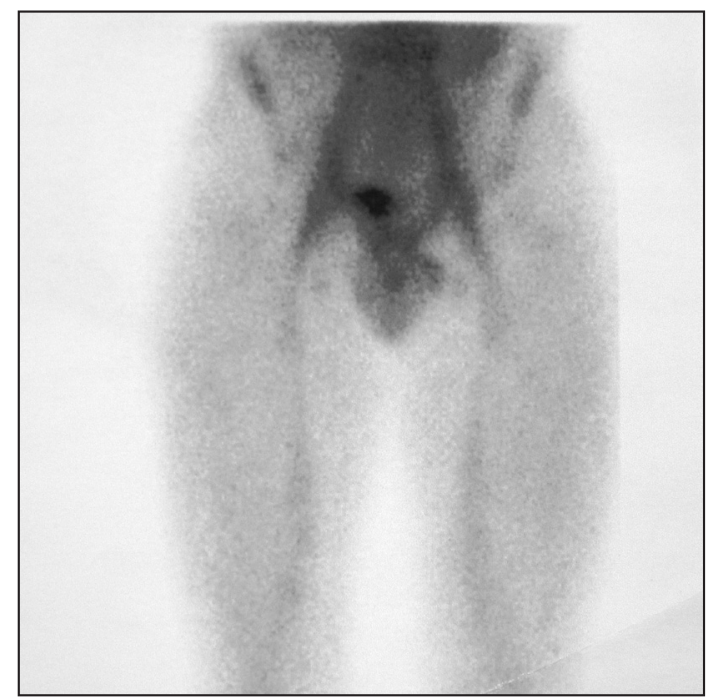

Figure 6 - Bone scintigraphy showing slight reuptake in the pubis, suggestive of bone remodeling.

then eccentric exercises to reinforce this musculature. The same was done in relation to the adductors. Hydrotherapy (whirlpool) work, bicycle exercises and gradually increasing load-bearing in the pool, with progressive movement, were performed. Subsequently, jumping exercises were performed in the pool.

Four weeks after the last MRI, the athlete was in the final phase of recovery, without any complaints. Proprioceptive hip exercises and functional exercises in the field were then started. Two weeks later, he was discharged by the medical department and he returned to sports practice without any limitations. Nonetheless, pelvic stabilization exercises and strengthening exercises for the adductor and abdominal musculature were expected to continue with the aim of avoiding recurrence of the injury.

\section{DISCUSSION}

Stress fractures of the pubic ramus are rare and usually occur in the medial portion or at the junction between the inferior pubic ramus and the ischium ${ }^{(1)}$. Its etiology is not very well delineated, but many factors (both extrinsic and intrinsic) are of importance in the evolution of bone injuries under stress ${ }^{(2)}$. There may be an imbalance in the relationship between osteogenesis and osteoclasis, which determines how physiological bone remodeling occurs, such that the responses to applied loads (compression, tension and spraining) are impaired, thereby generating continuity solutions in the bone tissue ${ }^{(2)}$. In the pubic bone, continuous tension from the adductor musculature, which originates at the junction between the inferior pubic ramus and the ischial ramus, is believed to be involved in the development of this injury. As the hip is extended, the pubic and ischial rami undergo intense lateral traction caused by the musculature, which is demonstrated by the anterolateral displacement of the bone fracture that is seen in avulsion fractures ${ }^{(1)}$.

In athletes, it is believed that weakening of the musculature reduces the shock absorption capacity of the lower limbs, thereby allowing redistribution of the forces on bones such that the stress on focal points in bones is increased. In physical overload situations, muscle fatigues would contribute towards evolution of stress fractures. Another possible explanation could be that the muscle traction through the bone might be capable of triggering sufficient repetitive force to trigger a bone failure ${ }^{(2)}$.

The age of the patient in our case needs to be mentioned because of its importance as a risk factor for stress fractures. Adolescents are believed to be more vulnerable to certain types of injury because of imbalance between strength and flexibility and the changes to the biomechanical properties of the bone that are inherent to the stretching that occurs through growth in height ${ }^{(5)}$. Among the extrinsic risk factors most present are the association between low levels of physical fitness and high volumes of training, and insufficient allowance of a rehabilitation period for previous injuries ${ }^{(2)}$.

In determining the diagnosis, in addition to the findings from the patient's history and clinical examination, the possibility of an avulsion fracture of 
the pubic ramus should be taken into consideration, because this is very common in adolescents. For our case, imaging methods were used, including radiography, MRI and bone scintigraphy.

The general principles of treatments for stress fractures can be applied to the pelvis ${ }^{(2)}$. Movements that might initially intensify the stress in the compromised region should be avoided, such as high-impact situations (running and jumping). Activities of daily living are maintained, including an early return to walking with weight-bearing on the limbs, from the outset of the treatment ${ }^{(2)}$. Physiotherapy should also aim to strengthen the abdominal and hip musculature, especially to avoid injury recurrence. The total duration of rehabilitation may have to be at least eight weeks ${ }^{(6)}$.

\section{REFERENCES}

1. Lee SW, Lee $\mathrm{CH}$. Fatigue stress fractures of the pubic ramus in the army: imaging features with radiographic, scintigraphic and MR imaging findings. Korean J Radiol. 2005;6(1):47-51.

2. Hosey RG, Fernandez MM, Johnson DL. Evaluation and management of stress fractures of the pelvis and sacrum. Orthopedics. 2008;31(4):383-5.

3. Snyder RA, Koester MC, Dunn WR. Epidemiology of stress fractures. Clin Sports Med. 2006;25(1):37-52.

4. Grava JPS, Pere AE, Camanho GL, Lasmar NP. Patologia do quadril na atividade esportiva. In: Lasmar NP, Camanho GL, Lasmar RCP. Medicina do Esporte. Rio de Janeiro: Revinter; 2000. p.313-8.

5. Shanmugam C, Maffulli N. Sports injuries in children. Br Med Bull. 2008;86:33-57.

6. Raasch WG, Hergan DJ. Treatment of stress fractures: the fundamentals. Clin Sports Med. 2006;25(1):29-36 